\title{
Fracturas metafisarias de radio distal desplazadas en el esqueleto en crecimiento: ¿cuál es el mejor método terapéutico? Revisión bibliográfica sistematizada
}

\author{
Displaced distal radius metaphyseal fractures in the growing skeleton: what is the \\ best therapeutic method? Systematized bibliographic review
}

\section{Fraturas metafisárias do rádio distal deslocadas no esqueleto em crescimento: qual é o melhor método terapêutico? Revisão bibliográfica sistematizada}

Mathias Maurente ${ }^{1}$, Ignacio Pagano1, Maria Elena Perez ${ }^{1}$

\section{RESUMEN}

Introducción: Las fracturas metafisarias de radio distal son las fracturas más frecuentes en la edad pediátrica, siendo 30\% del total de las mismas. Mantener la reducción de las fracturas desplazadas no siempre es posible: el re-desplazamiento es la principal complicación de estas lesiones. Clásicamente se realizó tratamiento con maniobra y yeso, aunque en los últimos años se asoció fijación con Kirschner wire (Kw) en búsqueda de disminuir el re-desplazamiento. El objetivo de nuestro trabajo es identificar factores de riesgo en búsqueda de realizar el mejor tratamiento siendo lo menos agresivo posible.

Materiales y métodos: Se realizó una búsqueda sistematizada a través del buscador electrónico PubMed. La misma alcanzó un total de 4594 artículos, que de acuerdo con los criterios de inclusión y exclusión se seleccionaron 15 trabajos para realizar nuestra revisión bibliográfica.

Resultados: Se destaca que la mayoría de los artículos encontrados son de nivel de evidencia III y IV. Nuestra búsqueda refleja un índice de re-desplazamiento entre $20-39 \%$ mediante yeso a las 2 semanas; asi como un indice de re-manipulación entre el 5-10 \% del total de las fracturas desplazadas. La mayoría de los estudios analizados intentan identificar los factores de riesgo más importantes para el re-desplazamiento de las fracturas, de lo que se destacan el grado de desplazamiento inicial de la fractura y la reducción lograda de la misma. A su vez, otros artículos valoran los resultados del tratamiento mediante estabilización con yeso o fijación mediante Kirschner wire.

Conclusión: el desplazamiento inicial de la fractura y la reducción lograda en block quirúrgico (BQ) son los factores de riesgo más importantes para el re-desplazamiento. Si bien la técnica de enyesado no es considerado una variable estadisticamente significativa, es considerada una variable importante en cuanto al pronóstico de la lesión, destacando al moldeado de 3 puntos como principal indice a considerar. Se considera aconsejable asociar un $\mathrm{Kw}$ en aquellas fracturas cabalgadas en las que no se logra una reducción anatómica en block quirúrgico.

\section{ABSTRACT}

Introduction: Distal radius metaphyseal fractures are the most frequent fractures in pediatric age, accounting for $30 \%$ of the total. Maintaining the reduction of displaced fractures is not always possible: re-displacement is the main complication of these injuries. Classically, treatment was performed with a maneuver and a cast. although in recent years fixation with Kirschner wire (Kw) has been associated in search of reducing re-displacement. The objective of our work is to identify risk factors in search of the best treatment while being the least aggressive possible.

Materials and methods: A systematic search was carried out using the PubMed electronic search engine. It reached a total of 4594 articles, which according to the inclusion and exclusion criteria, 15 papers were selected for our bibliographic review.

Results: It should be noted that most of the articles found are level of evidence III and IV. Our search reflects a re-displacement rate between 20-39\% using a cast at 2 weeks; as well as a remanipulation index between 5-10\% of all displaced fractures. Most of the studies analyzed attempt to identify the most important risk factors for the re-displacement of fractures, of which the degree of initial displacement of the fracture and the reduction achieved are highlighted. In turn, other articles assess the results of treatment by stabilization with plaster or fixation with Kirschner wire.

Conclusion: the initial displacement of the fracture and the reduction achieved in the surgical block $(\mathrm{BQ})$ are the most important risk factors for re-displacement. Although the casting technique is not considered a statistically significant variable, it is considered an important variable in terms of the prognosis of the injury, highlighting the 3-point casting as the main index to be considered. It is considered advisable to associate a Kw in those mounted fractures in which an anatomical reduction in surgical block is not achieved.

\section{RESUMO}

Introdução: As fraturas metafisárias do rádio distal são as mais frequentes na idade pediátrica, correspondendo a 30\% do total. Manter a redução das fraturas desviadas nem sempre é possivel: o deslocamento é a principal complicação dessas lesões. Classicamente, o tratamento era realizado com manobra e gesso, embora nos últimos anos a fixação com fio de Kirschner (KW) tenha sido associada na busca pela redução do deslocamento. O objetivo do nosso trabalho é identificar os fatores de risco em busca do melhor tratamento sendo o menos agressivo possivel.

Materiais e métodos: uma busca sistemática foi realizada usando o mecanismo de busca eletrônico PubMed. Chegou-se a um total de 4.594 artigos, que de acordo com os critérios de inclusão e exclusão, foram selecionados 15 artigos para nossa revisão bibliográfica.

Resultados: Ressalta-se que a maioria dos artigos encontrados são de nivel de evidência III e IV. Nossa pesquisa reflete uma taxa de re-deslocamento entre 20-39\% usando um gesso em 2 semanas; bem como um indice de remanipulação entre 5-10\% de todas as fraturas deslocadas. A maioria dos estudos analisados busca identificar os fatores de risco mais importantes para o deslocamento das fraturas, que incluem o grau de deslocamento inicial da fratura e a redução alcançada. Por sua vez, outros artigos avaliam os resultados do tratamento com estabilização gessada ou fixação com fio de Kirschner

Conclusão: o deslocamento inicial da fratura e a redução alcançada no bloqueio cirúrgico (QB) são os fatores de risco mais importantes para o deslocamento. Embora a técnica de gesso

1 Clinica de Traumatologia y Ortopedia Pediátrica "Prof. Dra. Maria Elena Pérez". Universidad de la República, Facultad de Medicina. Correo electrónico: mmaurente21@gmail.com, ORCID: 0000-0003-2216-6979

ORCID: 000-0003-4805-7543 
não seja considerada uma variável estatisticamente significativa, é considerada uma variável importante em termos de prognóstico da lesão, destacando-se o gesso em 3 pontos como o principal índice a ser considerado. Considera-se aconselhável associar um Kw nas fraturas montadas em que não se consegue redução anatômica no bloqueio cirúrgico.

\section{INTRODUCCIÓN}

Las fracturas de la extremidad distal del radio configuran la topografía más frecuente en la etapa del esqueleto inmaduro siendo el $20-30 \%$ del total de las mismas ${ }^{(1)}$. Las fracturas metafisarias son las más frecuentes, seguidas por las suprametafisarias y por último las fiso-epifisarias. Más del $80 \%$ de estas lesiones ocurren en pacientes mayores de 5 años, con un pico de incidencia que ronda los 10 años $^{(2,3,4)}$.

Tradicionalmente, la gran mayoría de estas lesiones eran tratadas de manera ortopédica mediante maniobra y yeso, lo que aún mantiene vigencia en el tratamiento, obteniendo entre 80 y $85 \%$ de resultados satisfactorios ${ }^{4}$ 5. 6). Las fracturas a este nivel tienen gran capacidad de consolidación y remodelación ósea, sobretodo en plano de movimiento y cuanto más próximas a la fisis sean; sin embargo múltiples estudios han demostrado que la remodelación completa no siempre se logra, especialmente en niños mayores de 8 años ${ }^{(4,6,7,8)}$. Mantener una reducción aceptable no siempre es posible, lo que puede generar re-desplazamiento o angulación de la fractura, que en ocasiones puede derivar en una re-manipulación de la fractura, en búsqueda de no obtener una consolidación viciosa de

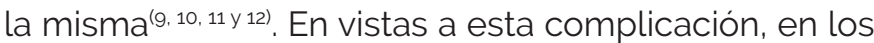
últimos años existe una tendencia a realizar una fijación con Kw, mediante diferentes técnicas $(7,13)$.

Este estudio intenta determinar los factores de riesgo de re-desplazamiento, valorando también los métodos de fijación, intentando dilucidar el mejor tratamiento para estas lesiones.

\section{OBJETIVOS GENERALES}

El objetivo de nuestro trabajo es revisar la evidencia acerca del tratamiento de las fracturas metafisarias de radio distal en el esqueleto en crecimiento valorando los resultados de los diferentes métodos terapéuticos.

\section{OBJETIVOS ESPECIFICOS}

Los objetivos especificos son valorar los factores de riesgo para el re-desplazamiento de las fracturas de puño, en búsqueda de acercarnos al mejor tratamiento siendo lo menos invasivo posible.

\section{MATERIALES Y MÉTODOS}

Se realizó en el mes de Octubre de 2019 la búsqueda bibliográfica sistematizada en los buscadores electrónicos PubMed y Science Direct. La búsqueda se realizó utilizando como término Mesh "Radial Fractures" y de manera manual agregamos "distal" y "treatment", ya que este término no está indexado como Mesh. Se utilizó para agrupar dichos términos el operador booleano "AND". Los filtros utilizados fueron artículos publicados entre 2009 y 2019, artículos en español e inglés, en pacientes hasta 18 años y en humanos.

\section{CRITERIOS DE INCLUSIÓN}

Se seleccionaron aquellos trabajos donde se incluian fracturas metafisarias de radio distal, en pacientes con esqueleto en crecimiento, en humanos, en inglés y español, realizados en los últimos 10 años.

\section{CRITERIOS DE EXCLUSIÓN}

Se excluyeron los trabajos sobre fracturas fisarias y suprametafisarias, asi como los trabajos de revisión bibliográfica y reportes de casos. No se excluyeron artículos por diferentes tiempos de seguimiento.

\section{ESTRATEGIA DE BÚSQUEDA}

Para la selección bibliográfica se utilizó como guía para el diagrama de flujo protocolo de revisiones PRISMA (Figura 1).

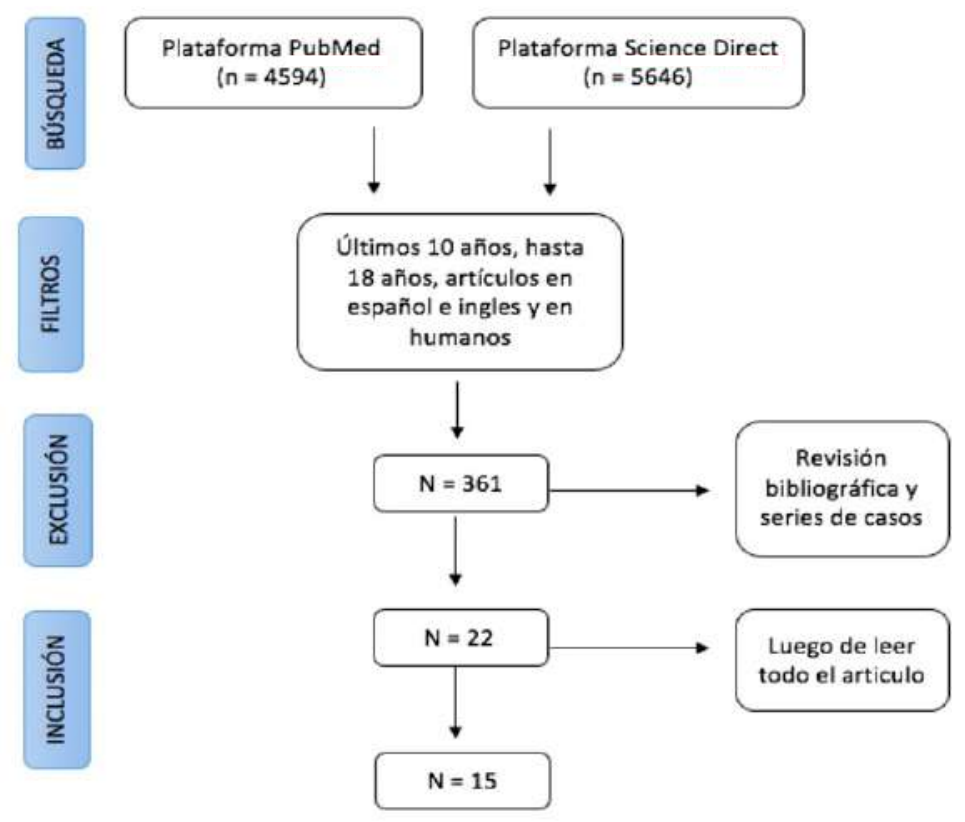

Figura 1.

Fuente: elaboración propia.

De la búsqueda inicial en las diferentes plataformas surgieron un total de 4594 artículos en Pubmed y 5646 en Science direct (total 10240).

Colocando los filtros mencionados, se obtuvieron 361 artículos entre ambas plataformas. Luego de leer 
los titulos, se seleccionaron 22 artículos, que luego de leerlos completamente, fueron excluidos 7 artículos, resultando finalmente en 15 trabajos para nuestro trabajo.

Realizada la búsqueda por dos revisores independientes, se llegó al mismo número de artículos.

De los 15 artículos incluidos, la mayoria son estudios retrospectivos, comparativos, con un nivel medio de evidencia: 3 y 4 (Figura 2).

\begin{tabular}{|c|c|c|c|}
\hline ARTÍCULO & $\begin{array}{l}\text { TIPO DE ESTUDIO Y } \\
\text { NIVEL DE EVIDENCIA }\end{array}$ & $\begin{array}{l}\text { NÜMERO DE } \\
\text { PACIENTES }\end{array}$ & SEGUIMIENTO \\
\hline Kamat ${ }^{(2)}$ & $\begin{array}{c}\text { Retrospectivo } \\
\text { comparativo. Nivel } 3 \text {. }\end{array}$ & 1001 & 2 semanas \\
\hline Jordan $^{(3)}$ & Retrospectivo. Nivel 4 & 105 & 6 semanas \\
\hline Satish ${ }^{(13)}$ & Retrospectivo. Nivel 4 & 52 & 1 año \\
\hline Jordan ${ }^{(15)}$ & Retrospectivo. Nivel 4 & 107 & 6 semanas \\
\hline Asadollahi ${ }^{(9)}$ & Prospectivo. Nivel 2 & 135 & 6 semanas \\
\hline Fenton ${ }^{(10)}$ & Retrospectivo. Nivel 3 & 73 & 6 semanas \\
\hline Hang ${ }^{(11)}$ & Retrospectivo. Nivel 3 & 662 & 2 meses \\
\hline McQuinn ${ }^{(15)}$ & Retrospectivo. Nivel 2 & 155 & 2 semanas \\
\hline Mazzini ${ }^{(16)}$ & Retrospectivo. Nivel 3 & 161 & 3 meses \\
\hline Sharma ${ }^{(12)}$ & Retrospectivo. Nivel 3 & 120 & 2 meses \\
\hline Ramoutar $^{(7)}$ & Retrospectivo. Nivel 3 & 248 & 7 semanas \\
\hline Wendling ${ }^{(17)}$ & Retrospectivo. Nivel 3 & 393 & 6 semanas \\
\hline Crawford ${ }^{(18)}$ & Prospectivo. Nivel 4 & 51 & 1 año \\
\hline Levy ${ }^{(19)}$ & $\begin{array}{l}\text { Prospectivo, } \\
\text { randomizado. Nivel } 2\end{array}$ & 72 & 3 meses \\
\hline Luscombe ${ }^{(8)}$ & Retrospectivo. Nivel 4 & 112 & 1 año \\
\hline
\end{tabular}

Figura 2.

Fuente: elaboración propia.

Los estudios analizados en su mayoría valoran posibles factores de riesgo para el re desplazamiento de la fractura; mientras que otros artículos valoran resultados mediante estabilización con yeso y/o Kirschner Wire $(\mathrm{Kw})$, ambos mediante diferentes técnicas.

De manera de poder analizar los resultados, se agruparon según su objetivo en 3 subgrupos:

1: 10 artículos que valoran factores de riesgo de desplazamiento;

2: 3 artículos que valoran fijación exclusivamente mediante yeso;

3: 2 artículos que valoran fijación y técnicas de reducción mediante Kw.

\section{RESULTADOS}

\section{FACTORES DE RIESGO}

Dentro de los artículos que valoran los distintos factores de riesgo para el re-desplazamiento de las fracturas, se observa que 9 de 10 artículos son estudios retrospectivos; dentro del cual se destaca el trabajo de Kamat et al. ${ }^{(2)}$ por su $\mathrm{N}$ de 1001 pacientes; aunque tiene únicamente 2 semanas de seguimiento. Por otro lado, el trabajo de Hang et al. ${ }^{(11)}$, cuenta con un N de 662 pacientes y 2 meses de seguimiento. Asadollahi et al. (9) es el único trabajo prospectivo en nuestra búsqueda, con un $\mathrm{N}$ de 135 y 6 semanas de seguimiento.
De nuestra búsqueda se desprende que múltiples autores han intentado identificar los factores de riesgo más importantes de re-desplazamiento, en búsqueda de disminuir el mismo y obtener mejores resultados. Los factores de riesgo que fueron valorados por la mayoría de los autores podrían dividirse según: tipo de fractura, paciente y método de estabilización. En cuanto al tipo de fractura las variables consideradas fueron: desplazamiento inicial, calidad de reducción intraoperatoria, dirección del trazo, fractura cubital asociada, cercanía a la fisis e intentos de reducción. En cuanto al paciente: edad y tumefacción. En referencia al método de estabilización: técnica de enyesado (distintos índices e inclusión del codo) y fijación con Kw (diferentes técnicas).

Si bien no todos los autores tomaron en cuenta todas las variables mencionadas, en todos los casos, se observó que el desplazamiento inicial de la fractura es un factor de riesgo significativo para el desplazamiento posterior de la misma, siendo el principal factor para la mayoria de los autores ${ }^{(3,8,9,11,12,14,15,16)}$ (Figura 3).

\begin{tabular}{|c|c|c|c|c|}
\hline ARTICULO & $\begin{array}{l}\text { DESPLAZAMIENTO } \\
\text { INICIAL }\end{array}$ & $\begin{array}{c}\text { REDUCCIÓN } \\
\text { INTRAOPERATORIA }\end{array}$ & $\begin{array}{c}\text { FRACTURA } \\
\text { CUBITAL } \\
\text { ASOCIADA }\end{array}$ & $\begin{array}{l}\text { TÉCNICA DE } \\
\text { ENYESADO }\end{array}$ \\
\hline Kamat $^{(2)}$ & Significativo & Significativo & $\begin{array}{c}\text { No } \\
\text { significativo }\end{array}$ & $\begin{array}{c}\text { Significativo } \\
\text { Cast Index }\end{array}$ \\
\hline Jordan ${ }^{(3)}$ & Significativo & Significativo & $\begin{array}{c}\text { No } \\
\text { significativo }\end{array}$ & $\begin{array}{c}\text { No } \\
\text { significativo }\end{array}$ \\
\hline Asadollahi $^{(9)}$ & Significativo & Significativo & $\begin{array}{c}\text { No } \\
\text { significativo }\end{array}$ & $\begin{array}{c}\text { No } \\
\text { significativo }\end{array}$ \\
\hline Hang (11) & Significativo & Significativo & Significativo & $\begin{array}{c}\text { No } \\
\text { significativo }\end{array}$ \\
\hline MCQuinn ${ }^{(15)}$ & Significativo & Significativo & $\begin{array}{c}\text { No } \\
\text { significativo }\end{array}$ & $\begin{array}{l}\text { Significativo } \\
3 \text { puntos }\end{array}$ \\
\hline Mazzini ${ }^{(16)}$ & Significativo & Significativo & $\begin{array}{c}\text { No } \\
\text { significativo }\end{array}$ & $\begin{array}{c}\text { No } \\
\text { significativo }\end{array}$ \\
\hline Sharma ${ }^{(12)}$ & Significativo & Significativo & $\begin{array}{c}\text { No } \\
\text { significativo }\end{array}$ & $\begin{array}{c}\text { No } \\
\text { significativo }\end{array}$ \\
\hline Ramoutar ${ }^{(7)}$ & Significativo & Significativo & $\begin{array}{c}\text { No } \\
\text { significativo }\end{array}$ & $\begin{array}{l}\text { Significativo } \\
3 \text { puntos }\end{array}$ \\
\hline Luscombe $^{(a)}$ & Significativo & Significativo & $\begin{array}{c}\text { No } \\
\text { significativo }\end{array}$ & $\begin{array}{c}\text { No } \\
\text { significativo }\end{array}$ \\
\hline Fenton ${ }^{(10)}$ & Significativo & Significativo & $\begin{array}{c}\text { No } \\
\text { significativo }\end{array}$ & $\begin{array}{c}\text { No } \\
\text { significativo }\end{array}$ \\
\hline Jordan ${ }^{(14)}$ & Significativo & Significativo & Significativo & $\begin{array}{c}\text { No } \\
\text { significativo }\end{array}$ \\
\hline Wendling ${ }^{(17)}$ & Significativo & Significativo & $\begin{array}{c}\text { No } \\
\text { significativo }\end{array}$ & $\begin{array}{c}\text { No } \\
\text { significativo }\end{array}$ \\
\hline
\end{tabular}

Figura 3.

Fuente: elaboración propia.

El siguiente factor de riesgo es la calidad de la reducción lograda en el intraoperatorio. La mayoría de los autores se refiere a reducción anatómica como aquella reducción con menos de 10\% de traslación y menos de $5 \%$ de angulación en cualquier sentido ${ }^{3}$. 14). En su artículo, Jordan et al.(3) refiere que logrando una reducción anatómica, ninguna fractura sufrió desplazamiento posterior; mientras que aquellas con una reducción sub-óptima, 40\% si lo sufrieron. A su vez, Asadollahi et al. ${ }^{(9)}$ refleja que la reducción anatómica es un factor protector significativo para el desplazamiento posterior, así como Mazzini et al.(17) manifiesta que la reducción anatómica es el factor pronóstico más importante: reducción no anatómica aumenta 5 veces más chances de desplazamiento posterior. 
En cuanto a la fractura cubital asociada, únicamente los autores RW Jordan ${ }^{(14)}$ y Hang et al. ${ }^{(11)}$ obtuvieron diferencias significativas: aumento del desplazamiento posterior si asociaba dicha fractura. En el resto de artículos que estudiaban dicha variable no encontraron diferencias significativas $(9,10,12,16,17)$. Asi como tampoco se encontró evidencia de que alguna dirección o tipo de trazo de fractura en el radio se asocie de forma significativa con el re desplazamiento.

Por otro lado, Wendling et al. ${ }^{(18)}$ fue el único autor que valoró la necesidad de realizar múltiples intentos de reducción en el mismo acto: observaron que si la reducción de la fractura resultaba dificultosa, la misma presentaba mayor riesgo de desplazamiento posterior. Mientras que en aquellas fracturas sin dificultades en su reducción: $18,5 \%$ se desplazaron, el $45 \%$ sufrieron re desplazamiento si la reducción de la fractura era dificultosa.

En cuanto a las variables correspondiente al paciente, la edad fue una variable estudiada por la gran mayoría de los autores, observándose una edad media de 10 años. Sin embargo solo Mazzini et al.(17) obtuvo diferencias significativas: dicho estudio reflejó que los pacientes mayores de 14 años sufrían mayor tendencia a desplazarse bajo yeso.

Sobre el método de estabilización, se valoraron principalmente 2 opciones: yeso (BP o ABP) o fijación con Kw asociada a férula ABP. De nuestra bibliografia se destacan dos artículos que utilizan ambos métodos, de forma retrospectiva. Por un lado, Lascombe et al (8) refiere no observar re-desplazamiento en aquellas fijadas con Kw, aunque unicamente utiliza Kw en 7 fracturas. Por otro lado, RW Jordan et al. ${ }^{(3)}$ utiliza Kw en 17 fracturas y yeso en 56 fracturas. En aquellas fracturas estabilizadas con Kw, ninguna sufrió redesplazamiento, mientras que 12 de las 56 fracturas estabilizadas con yeso sufrieron re-desplazamiento. Sin embargo, RW Jordan et al. destaca como variable significativa la reducción inicial y la reducción intraoperatoria en aquellos pacientes estabilizados con yeso.

Múltiples artículos registraron los resultados de la inmovilización con yeso; valorando a su vez distintos indices en busca de objetivar la técnica de enyesado y un pronóstico de desplazamiento según la calidad del aparato enyesado.

En cuanto a la inclusión del codo, los autores Asadollahi ${ }^{(9)}$, Fenton ${ }^{(10)}$ y Ramoutar ${ }^{(7)}$, no encontraron diferencias de desplazamiento posterior bajo yeso entre aquellos con yeso BP y ABP. Sin embargo, Kamat $^{(2)}$ y Hang ${ }^{(11)}$, observaron como factor de riesgo un Cast Index mayor a 0,7 (Figura 6); por su lado Jordan et al. ${ }^{(15)}$, destacó una tendencia pero no fue una variable significativa. En su artículo, Kamat ${ }^{(2)}$, valora los resultados de 1001 pacientes con fractura desplazadas de puño, reducidas en $B Q$, estabilizadas con yeso braqui-palmar (BP). 10,6\% sufrieron un desplazamiento mayor al aceptado y realizándose nueva maniobra; de esos 10,6\%, 75\% tenían un yeso con un $\mathrm{Cl}$ mayor a 0.8 . En los pacientes con un IC menor a 0,8, el índice de desplazamiento fue de $5 \%$, mientras que los $\mathrm{Cl}$ mayor a 0,8 sufrieron un $26 \%$ de desplazamiento bajo yeso. Este estudio también evidenció que cada 0,1 que aumentaba el $\mathrm{Cl}$ sobre 0,8 , el desplazamiento aumenta 3 grados promedialmente. Por otro lado, en su artículo Asadollahi et al.(9), destaca que no encontró diferencias significativas en cuanto al $\mathrm{Cl}$.

En su artículo, Hang et al. ${ }^{(11)}$, valoró también otros indices, además del Cl: el Gap Index y los 3 puntos de fijación, observando que en aquellos moldeados de yeso en que se logran los 3 puntos, este era un factor protector ante el desplazamiento bajo yeso. En sus artículos, McQuinn ${ }^{(16)}$ y Ramoutar(7), destacan también el indice de 3 puntos como el índice de enyesado más influyente, asi como un factor protector significativo.

\section{FIJACIÓN MEDIANTE YESO}

De nuestra búsqueda se desprende que en los artículos que utilizan únicamente yeso en su tratamiento, se valoran distintas técnicas de enyesado, valorando índices y resultados.

En su artículo Kamat ${ }^{(2)}$, con 1001 pacientes, valora principalmente el "Cast Index" destacando que cuando este es menor a 0,8, el indice de desplazamiento fue de $5 \%$, mientras que los $\mathrm{Cl}$ mayor a 0,8 sufrieron un $26 \%$ de desplazamiento bajo yeso.

Por su parte Crawford et al. ${ }^{(18)}$, realizó un estudio con 51 paciente con una edad media de 7 años, en el cual realiza yeso ABPen puerta de emergencia, sin sedación, corrigiendo únicamente angulación. El promedio de acortamiento inicial fue de $5 \mathrm{~mm}$, una angulación en frente de 4 grados y 3,2 en el perfil. Se mantuvo yeso 42 dias en promedio y se realizó seguimiento a la semana, dos semanas, 6 semanas y al primer año. Obtuvo una angulación promedio de 2,2 grados en plano sagital y 0,8 grados en plano coronal al final del tratamiento, con un rango completo de movilidad de puño.

Por otro lado, Levy et al.(19) realiza un estudio prospectivo, randomizado, en el cual compara yeso BP cerrado vs férula Bi-valva (una palmar y otra dorsal) braqui-palmar; con una inmovilización promedio de 7 semanas. Se incluyeron únicamente pacientes requirieron reducción en $B Q$ o en sala de emergencia. Se obtuvieron mejores resultados en el grupo inmovilizado con férula durante el periodo de inmovilización, sin embargo no se observaron diferencias significativas al momento de retiro de inmovilización.

\section{FIJACIÓN MEDIANTE KIRSCHNER}


A cerca de la fijación utilizando alambres de Kirschner (Kw), múltiples estudios han estudiado sus indicaciones, resultados y complicaciones. De nuestra búsqueda se desprende que los autores utilizan los Kw como método de fijación cuando la fractura tiene un desplazamiento inicial importante (menos de $50 \%$ de contacto), cuando no se logra una buena reducción intra-operatoria, fijación de fractura que sufrió desplazamiento bajo yeso, cuando el paciente tiene una tumefacción considerable y como método de reducción simil técnica de Kapandji. En la gran mayoría de los artículos la fijación con Kw disminuyó los porcentajes de desplazamiento en comparación con yeso(4, 8,16).

En cuanto a las complicaciones, en sus artículos Satish ${ }^{(13)}$, Hang ${ }^{(11)}$, Sharma ${ }^{(12)}$ y Luscombe ${ }^{(8)}$ no observaron ninguna complicación utilizando Kw, en un total de 170 pacientes enclavijados con Kw. Sin embargo, Ramoutar et al. $^{(7)}$, en su artículo con 112 pacientes, reportó complicaciones en el $17 \%$ de los pacientes. Estas complicaciones fueron: migración de Kw hacia el hueso con necesidad de cirugia para retirarlos, granulación excesiva que requirió tratamiento, infección superficial que requirió antibióticos, nueva cirugía por desplazamiento, migración hacia afuera del Kw y neuroapraxia del nervio cubital.

En cuanto a los autores que realizan fijación primaria con alambres, Satish et al. ${ }^{(13)}$ utiliza Kw a los 52 pacientes de su estudio, todos con fracturas

metafisarias desplazadas a dorso. Este autor utiliza los Kw como método de reducción intrafocal, simil técnica de Kapandji y luego lo fija. Refiere obtener muy buenos resultados, sin desplazamiento posterior, ni otras complicaciones asociadas a los Kw.

En su artículo de 248 pacientes, Ramoutar et al. ${ }^{(7)}$, realizó un estudio comparativo randomizado entre fracturas desplazadas, en las que estabilizó mediante yeso o Kw.

Se destaca que este autor utiliza técnica de fijación transestiloideo. El autor refiere que no encontró diferencias significativas en cuanto al número de Kw necesarios para inmovilizar la fractura; se trataron 162 pacientes con 1 Kw, 82 con 2 y 4 con 3kw (Figura 4).

\section{DISCUSIÓN}

En primer lugar, de nuestra revisión se desprende que, si bien las fracturas de radio distal son una patología sumamente frecuente, aún existe controversia en cuanto al mejor tratamiento: lograr los mejores resultados siendo lo menos invasivo posible.

En la bibliografia, se destaca como principal complicación de estas fracturas el re-desplazamiento. En nuestra búsqueda observamos que si bien el porcentaje de re-desplazamiento en pacientes inmovilizados con yeso se encontró entre 21 y 39\%(2, 3. 9, $10,12,14,15,16,17)$, el criterio para definir el re desplazamiento no fue el mismo en todos los artículos. Por lo general se interpretó como desplazamiento bajo yeso una angulación mayor a 10 grados en cualquier sentido en las primeras dos semanas 2 (2, 3, 9, 15, 16).

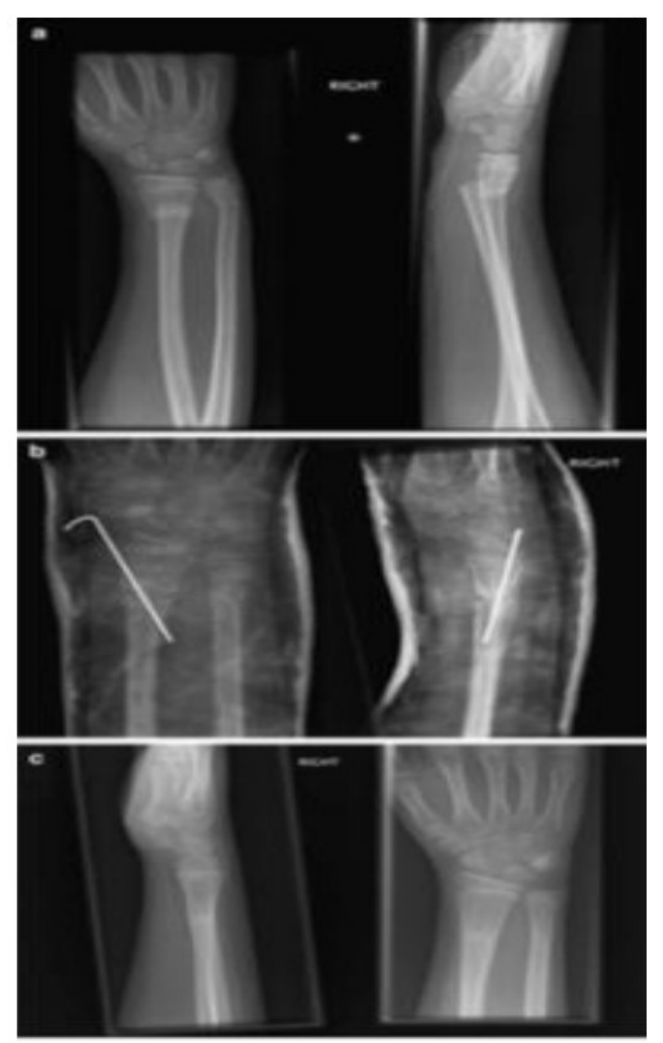

Figura 4. Fractura desplazada tratada inicialmente con Kw Fuente: elaboración propia.

En cuanto a la re manipulación por re-desplazamiento, observamos que el porcentaje del mismo osciló entre el 5 a $10 \%(2,9,10,11,12,14,15)$. Sin embargo no todos los autores compartieron el grado de desplazamiento que justifique realizar una nueva maniobra de reducción. Por ejemplo, en su trabajo Amaya et al. ${ }^{(2)}$ describe que se realizó una manipulación en block quirúrgico en pacientes con más de 15 grados de desplazamiento y/o más de $80 \%$ de traslación, mientras que, en sus artículos, Jordan et al.(3,14) admiten hasta 20 grados de angulación o menos de 50\% de contacto óseo.

Por otro lado Hang et al. (11) realizó re manipulación en aquellos pacientes que presentaban deformidad clínica evidente y sin signos de consolidación en la radiografía. En los casos en los que se realizó una remanipulación en block quirúrgico, se asoció Kw en la mayoría de los casos (Figura 5).

Por otro lado, del presente trabajo se evidencia que existen 2 factores de riesgo que se destacan significativamente sobre los otros: desplazamiento inicial de la fractura y la reducción anatómica. Los trabajos demuestran que se ha logrado disminuir el 


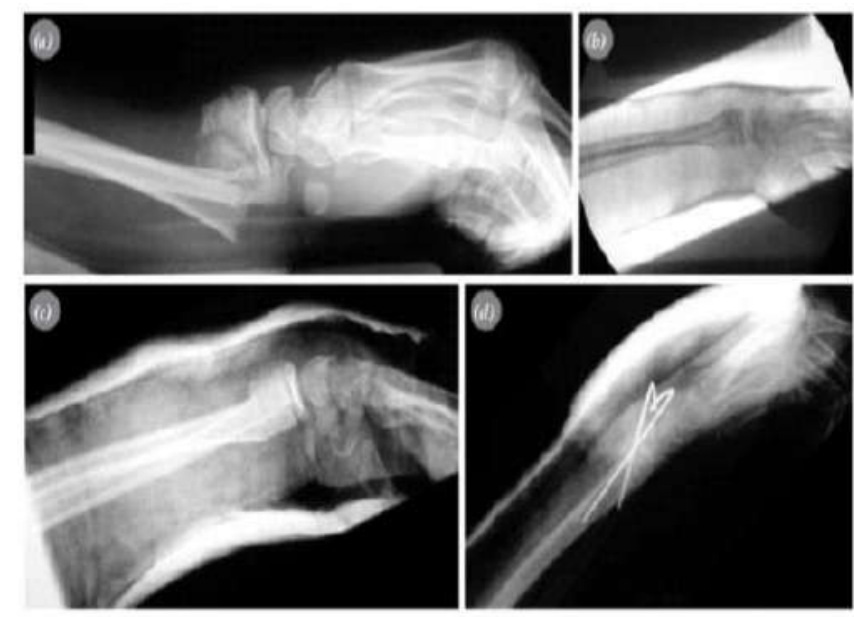

Figura 5. Paciente sufre re desplazamiento que requiere re manipulación y fijación con Kw

Fuente: elaboración propia.

re-desplazamiento mediante la colocación de $\mathrm{Kw}^{13 .}$ 5. 6), con mínimo porcentaje de complicaciones ${ }^{(3,8,11,}$ 12. 13). En su artículo, Zeng et al.(4) describe un $40 \%$ de re-desplazamiento en los pacientes tratados con yeso, mientras que únicamente $3,6 \%$ lo sufrió con fijación Kw. Las razones por las cuales se cree que el desplazamiento inicial es un factor importante para el pronóstico son: lesión de la bisagra perióstica, mayor desplazamiento lleva a mayor tumefacción y componente secundario, generando mayor posibilidad de desplazamiento bajo yeso. La reducción anatómica, es factor protector contra el desplazamiento posterior, tiende a darse menos frecuentemente cuanto mayor sea el desplazamiento inicial.

En cuanto a la técnica de enyesado, podemos evidenciar que se toman en cuenta múltiples indices (Figura 6), siendo el más significativo en vistas al resultado el moldeado de 3 puntos ${ }^{(7,15)}$, no existiendo consenso en la bibliografia si es un factor significativo en cuanto al desplazamiento posterior.

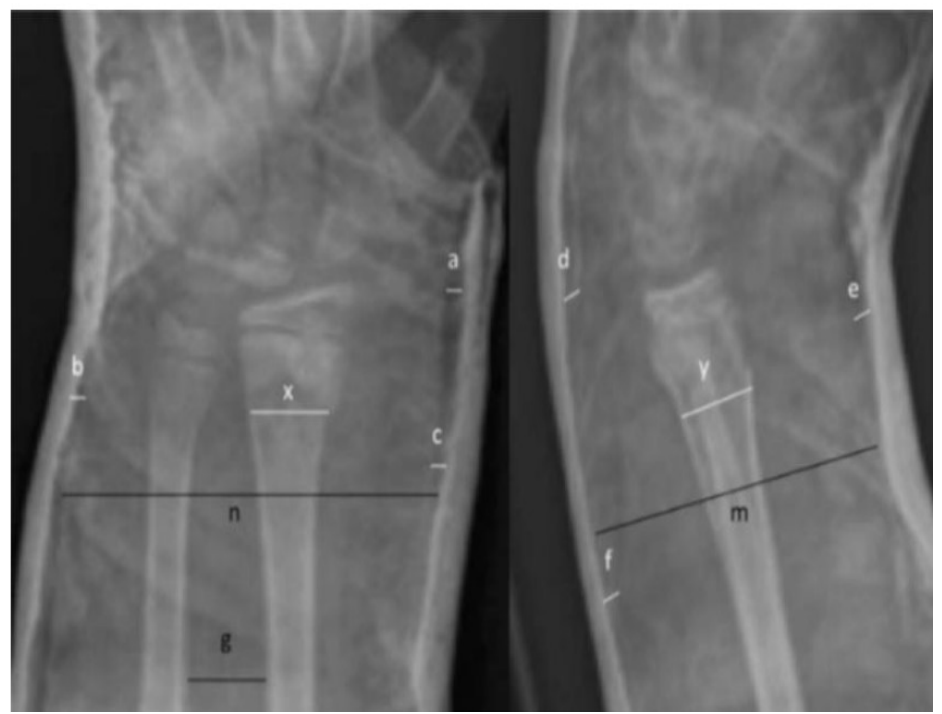

Figura 6. 3 puntos $=(a+b+c) / x+(c+d+e) / y$. Padding index $=e / g$. Cast Index $=\mathrm{m} / \mathrm{n}$. Canterbury index $=$ Padding index + cast index

Fuente: elaboración propia.
Destacamos que en nuestra revisión nos encontramos con algunas limitaciones. Los estudios tienen relativamente poco tiempo de seguimiento, lo cual es fundamental para evaluar el resultado final del tratamiento. Si bien Ramoutar et al. ${ }^{(7)}$ refiere que observó una función limitada si el paciente presentaba 15 grados de angulación al comenzar movilidad, los trabajos no cuentan con un score que valore la funcionalidad de la articulación del puño a corto y largo plazo. Creemos que conocer los resultados funcionales de las fracturas metafisarias desplazadas de radio distal resulta fundamental a la hora de valorar la necesidad de realizar una intervención más agresiva de forma inicial o que justifique realizar una re-manipulación en la evolución, teniendo en cuenta el potencial de remodelación en los pacientes con esqueleto en crecimiento.

\section{CONCLUSIONES}

Hemos revisado de la bibliografia actual cual es el mejor tratamiento para las fracturas de puño en el esqueleto inmaduro. Creemos es un trabajo de gran interés para el ortopedista, ya que, es una patología sumamente común que no logra excelentes resultados en la totalidad de los casos.

De la bibliografía analizada podemos concluir que el desplazamiento inicial de la fractura y la reducción lograda en $B Q$ son los factores de riesgo más importantes para el re-desplazamiento. La técnica de enyesado es un elemento importante a tener en cuenta, en el cual el moldeado de 3 puntos es el principal indice a considerar. Sería aconsejable asociar un Kw en aquellas fracturas cabalgadas en las que no se logra una reducción anatómica en BQ. Destacamos que existe poco nivel de evidencia para el mejor tratamiento de estas lesiones, asi como artículos con seguimiento a largo plazo.

\section{REFERENCIAS}

1. Waters P. Fracturas del cubito y radio distal. En: Rockwood y Wilkins, ed. Fracturas en el niño. 5a ed. Madrid: Marban; 2007. p. $381-442$.

2. Kamat AS, Pierse N, Devane P, Mutimer J, Horne G. Redefining the Cast index: the optimum technique to reduce redisplacement in pediatric distal forearm fractures. J Pediatr Orthop. 2012:32(8):787-791

3. Jordan J, Westacott DJ. Displaced paediatric distal radius fractures-When should we use percutaneous wires? Trauma \& Orthopaedic Department, Birmingham Heartlands Hospital, United Kingdom. 2012; 908-911

4. Zhi-Kui Zeng MD, Wei-Dong Liang MM, You-Qiang Sun MD, Ping-Pin Jiang BS, Ding Li MD, Zhen Shen MD, et al. Is percutaneous pinning needed for the treatment of displaced distal radius metaphyseal fractures in children? A systematic review. Medicine. 2018:97:36

5. Pretell Mazzini J, Rodriguez Martin J. Paediatric forearm and distal radius fractures: risk factors and re-displacement- 
role of casting indices. International Orthopaedics (SICOT). 2010;34:407-412

6. Miller BS, Taylor B, Widmann RG, Bae DS, Snyder BD, Waters PM. Cast Immobilization Versus Percutaneous Pin Fixation of Displaced Distal Radius Fractures in Children A Prospective. Randomized Study. J Pediatr Orthop. 2005;25(4):490-494

7. Ramoutar DN, Shivji FS, Rodrigues JN, Hunter JB. The outcomes of displaced paediatric distal radius fractures treated with percutaneous Kirschner wire fixation: a review of 248 cases. Eur J Orthop Surg Traumatol. 2015:25(3):471-6

8. Luscombe KL, Chaudhry S, Dwyer JSM, Ssanmuganm C, Maffuli N. Selective Kirschner wiring for displaced distal radial fractures in children. Acta Orthop Traumatol Turc. 2010; 44(2): 117-123.

9. Asadollahi S, Ooi KS, Hau RC. Distal Radial Fractures in Children Risk Factors for Redisplacement Following Closed Reduction J Pediatr Orthop. 2015:35(3):224-228

10. Fenton $\mathrm{P}$, Nightingale $\mathrm{P}$, Hodson J, Luscombe J. Factors in redisplacement of paediatric distal radius fractures. J Ped Orthop. 2012;21:127-130

11. Hang JR, Hutchinson AF, Hau RC. Risk Factors Associated With Loss of Position After Closed Reduction of Distal Radial Fractures in Children. J Pediatr Orthop. 2011:31:501-506

12. Sharma S, Bowe D, Walters SJ, Flowers MJ. Dorsal cortical comminution as a predictor of redisplacement of distal radius fractures in children. Injury, Int. J. Care Injured. 2011;421:173177

13. SatiSh BRJ, MS, Vinodkumar M, Suresh M, Seetharam P, Jaikumar K. Closed Reduction and K-Wiring With the Kapandj Technique for Completely Displaced Pediatric Distal Radial Fractures. Orthopedics. 2014:37(9):e810-6

14. Jordan RW, Westacott D, Srinivas K, Shyamalan G. Predicting redisplacement after manipulation of paediatric distal radius fractures: the importance of cast moulding. Eur J Orthop Surg Traumatol. 2015;25(5):841-5

15. McQuinn AG, Phys B, Jaarsma RL. Risk Factors for Redisplacement of Pediatric Distal Forearm and Distal Radius Fractures. Pediatr Orthop 2012;32:687-692

15. Pretell Mazzini J, Beck N, Brewer J, Baldwin K, Sankar W, Flynn J. Distal metaphyseal radius fractures in children following closed reduction and casting: can loss of reduction be predicted? International Orthopaedics (SICOT). 2012;36:14351440

16. WendlingKeim DS, Wieser B, Dietz HG. Closed reduction and immobilization of displaced distal radial fractures. Method of choice for the treatment of children? Eur J Trauma Emerg Surg. 2015:41(4):421-8

17. Crawford SM, Lee LSK, Izuka BH. Closed Treatment of Overriding Distal Radial Fractures without Reduction in Children. J Bone Joint Surg Am. 2012:94:246-52

18. Levy J, Ernat J, Song D, Cook JB, Judd D, Shaha S. Outcomes of Long-arm Casting Versus Double-Sugar- Tong Splinting of Acute Pediatric Distal Forearm Fractures. J Pediatr Orthop. 2015:35(1):11-17

Nota del autor:

Los autores declaran no tener ningún conflicto de intereses.

\section{Nota del Editor:}

El presente manuscrito fue aprobado por los Dres. Asdrúbal Silveri y Enrique Barrios

\section{Nota de contribución:}

Dr. Mathias Maurente

A) Revisión bibliográfica sistematizada

También realizo B, C, D, E y F.

Dr. Ignacio Pagano

A) Revisión bibliográfica sistematizada

B) recolección de datos

G) Supervisión del trabajo

Dra. M. E. Perez

G) Supervision del trabajo 